\title{
Condições para desidratação osmótica de laranjas e as propriedades funcionais do produto
}

\author{
Gabriela R. L. Mendes ${ }^{1}$, Cristina H. de Freitas ${ }^{1}$, Priscila T. Scaglioni ${ }^{2}$, \\ Cristiano G. Schmidt ${ }^{3}$ \& Eliana B. Furlong ${ }^{2}$
}

\begin{abstract}
RESUMO
Neste trabalho foram estudadas as melhores condições para a desidratação osmótica de laranjas, sendo verificadas as alterações nas características físico-químicas da fruta. Um planejamento experimental fatorial $2^{3}$ completo foi realizado, analisando-se a influência dos fatores tempo, da concentração da solução e proporção fruta:solução osmótica sobre o ganho de sólidos, a perda de água e redução de massa das laranjas desidratadas osmoticamente. Após desidratação, as frutas foram secas em secador de bandejas até umidade de $20 \%$. Determinaram-se no produto, o pH, a acidez titulável, a vitamina C, os carotenoides, o conteúdo fenólico e a cor. Os melhores resultados de desidratação osmótica foram obtidos utilizando-se uma solução de sacarose de $60^{\circ}$ Brix, na proporção fruta:solução de 1:3 durante 90 min, que resultou em

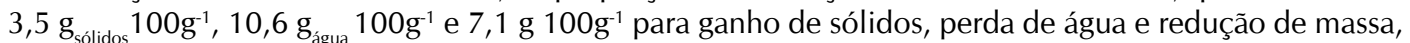
respectivamente. As perdas de conteúdo fenólico e a atividade antioxidante foram 45,1 e 37,3\% menores que na secagem convencional demonstrando a eficiência do processo para preservação das propriedades funcionais do produto.
\end{abstract}

Palavras-chave: Citrus sinensis, secagem, vitamina C, compostos fenólicos, planejamento experimental

\section{Conditions for osmotic dehydration of oranges and functional properties of the product}

\begin{abstract}
The best conditions for osmotic dehydration of oranges was studied, assessed by determining the physicochemical characteristics of the fruit. The study was conducted in $2^{3}$ full factorial experimental design to evaluate the influence of time, solution concentration and fruit:solution ratio on the solid gain, water loss and mass reduction of osmotically dehydrated oranges. After dehydration, the fruits were dried in tray dryer until $20 \%$ moisture. In the product the $\mathrm{pH}$, titratable acidity, vitamin $\mathrm{C}$, carotenoids, phenols content and color were determined. The best results of osmotic dehydration were obtained using a sucrose solution 60

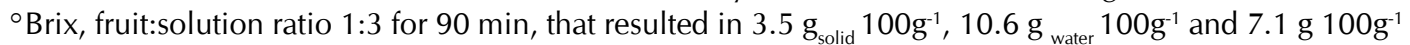
of solid gain, water loss and weight reduction, respectively. The losses of phenolic content and antioxidant activity were 45.1 and $37.3 \%$ lower than in conventional drying, demonstrating the efficiency of the process for the preservation of functional properties of the product.
\end{abstract}

Key words: Citrus sinensis, drying, vitamin C, phenolic compounds, experimental design

UFPel, Campus Universitário Capão do Leão, S/N, CEP 96900-010, Capão do Leão, RS. Fone: (53) 8111-2038 E-mail: gabrielalemosmendes@gmail.com; crishallal@hotmail.com

${ }^{2}$ FURG, Campus Cidade, Rua Engenheiro Alfredo Huch, 475, Bairro Santa Tereza, CEP 96200-970, Rio Grande, RS. Fone: (53) $3233-8663$. E-mail: priscilascaglioni@gmail.com; dqmebf@furg.br

${ }^{3}$ FURG, Campus Santo Antônio da Patrulha, Rua Barão do Caí, 125, Bairro Cidade Alta, CEP 95500-000, Santo Antônio da Patrulha, RS Fone: (51) 3662-7803. E-mail:cristiano@furg.br 


\section{INTRODUÇÃO}

A produção mundial de laranjas excedeu 60 milhões de toneladas nos últimos anos sendo que o Brasil é responsável por $30 \%$ desta produção. São Paulo é o estado que mais contribui para este índice; segundo o CONAB, a estimativa da área em produção é de 525.514 mil ha. O cultivar da laranja Valência é economicamente importante devido à alta produtividade $\mathrm{e}$ qualidade das frutas, apresentando maturação tardia e podendo ser destinada tanto para o mercado interno como para o externo, atendendo ao consumo da fruta fresca e ao processamento industrial (Tazima et al., 2008; CONAB, 2012).

Com o aumento da exigência dos consumidores por alimentos naturais e saudáveis, produtos à base de frutas devem ser processados de maneira a preservar as características sensoriais e, principalmente, as propriedades funcionais; no caso da laranja essas propriedades estão relacionadas ao conteúdo de carotenoides e vitamina C (Carvalho et al., 2006; Branco et al., 2007). Técnicas de processamento que propiciam a redução de água livre sob condições brandas, como a desidratação osmótica, podem ser uma alternativa viável com vista à obtenção de produtos de alta qualidade com uma vida longa de prateleira, além de prevenir alterações indesejáveis de cor, aroma, textura e sabor, que ocorrem durante o armazenamento (Rodrigues et al., 2008).

A desidratação osmótica é uma operação de transferência de massa em que a água é parcialmente removida dos alimentos por imersão em soluções aquosas concentradas, tais como xaropes ou salmouras. Durante o processo ocorrem incorporação de sólidos e consequente diminuição da atividade de água e diminuição da velocidade de deterioração do alimento (Martim et al., 2007; Ruiz-López et al., 2011). As vantagens desta operação consistem em diminuir o teor de umidade inicial do alimento, conservar as características sensoriais e nutricionais da fruta e reduzir o tempo de secagem convectiva e o custo do processo. A desvantagem é a permanência de uma fina camada de açúcar, indesejável na superfície da fruta e, dependendo do tipo de fruta, pode tornarse rançosa e com teor de umidade muito baixo (Medeiros et al., 2006; Demczuk Júnior et al., 2008).

A qualidade da desidratação osmótica depende de diferentes fatores, como o agente desidratante e sua concentração, a temperatura, o tempo de imersão, a natureza dos frutos e da superfície exposta à troca osmótica (Tsamo et al., 2005; Eren \& Kaymak-Ertekin, 2007; Dionello et al., 2009). A laranja, além de ser uma fruta amplamente consumida, desperta o interesse devido ao seu alto teor de vitamina $\mathrm{C}$ e compostos fenólicos, motivo pelo qual são necessários estudos de técnicas que permitam a conservação desses compostos e também aumentem a vida útil do produto que pode ser destinado para o consumo direto ou utilizado como ingrediente em formulações alimentícias. Neste estudo foram determinadas as melhores condições experimentais para desidratação osmótica de laranjas avaliadas através da determinação das características físico-químicas da fruta.

\section{Material e Métodos}

A matéria-prima utilizada na operação de desidratação osmótica foi a laranja da variedade Valência, proveniente de um único produtor, para minimizar o efeito da heterogeneidade da amostra. Após serem devidamente higienizadas as laranjas foram descascadas e cortadas manualmente, em pedaços circulares de $1 \mathrm{~cm}$ de espessura e $6 \mathrm{~cm}$ de diâmetro.

A operação de desidratação osmótica foi realizada em agitador horizontal a $70 \mathrm{rpm}$ a $20^{\circ} \mathrm{C}$, utilizando sacarose como agente osmótico em diferentes tempos de desidratação. Após a desidratação as amostras foram submetidas a jatos de água destilada em ambos os lados para retirar o excesso de soluto na superfície da fruta; o excesso de líquido da superfície foi removido com papel absorvente. Para avaliar a eficiência da operação de desidratação osmótica foram determinados o ganho de sólidos (GS), a perda de água (PA) e a redução de massa (RM) calculados conforme as Eqs. 1, 2 e 3, respectivamente (Eren \& Kaymak-Ertekin, 2007; Dionello et al., 2009).

$$
\begin{gathered}
\mathrm{GS}=\frac{\mathrm{M}_{\mathrm{i}}-\mathrm{M}_{\mathrm{f}}}{\mathrm{M}_{\mathrm{i}}} \times 100 \quad\left(\mathrm{~g}_{\text {sólidos }} 100 \mathrm{~g}^{-1}\right) \\
\mathrm{PA}=\frac{\left(\mathrm{M}_{\mathrm{i}} \times \mathrm{X}_{\mathrm{i}}\right)-\left(\mathrm{M}_{\mathrm{f}} \times \mathrm{X}_{\mathrm{f}}\right)}{\mathrm{M}_{\mathrm{i}}} \times 100 \quad\left(\mathrm{~g}_{\text {agua }} 100 \mathrm{~g}^{-1}\right) \\
\mathrm{RM}=\mathrm{PA}-\mathrm{GS} \quad\left(\mathrm{g} 100 \mathrm{~g}^{-1}\right)
\end{gathered}
$$

em que:

$\mathrm{M}_{\mathrm{i}} \quad$ - massa inicial de laranja in natura, $\mathrm{g}$

$\mathrm{M}_{\mathrm{F}}$ - massa da laranja após desidratação osmótica, $\mathrm{g}$

$\mathrm{X}_{\mathrm{i}} \quad$ - fração de água da laranja in natura

$\mathrm{X}_{\mathrm{F}} \quad$ - fração de água da laranja após desidratação osmótica

Para estabelecer as melhores condições na operação de desidratação osmótica foi utilizado um planejamento experimental fatorial $2^{3}$ completo e avaliados o efeito do tempo de desidratação, a concentração da solução e a proporção fruta:solução osmótica sobre o GS, a PA e a RM das laranjas desidratadas osmoticamente. A Tabela 1 apresenta os níveis e os valores estudados de cada variável cujos resultados foram tratados por análise de efeitos e de superfície de resposta através do software Statistica 7.0. Foram geradas superfícies de respostas para as variáveis que influenciaram os parâmetros estudados.

Tabela 1. Níveis das variáveis independentes e valores de tempo, concentração da solução e proporção fruta:solução usados no planejamento experimental para desidratação osmótica de laranjas

\begin{tabular}{lccccc}
\hline \multirow{2}{*}{ Fatores } & \multicolumn{5}{c}{ Níveis } \\
\cline { 2 - 6 } & $-\boldsymbol{\alpha}$ & $-\mathbf{1}$ & $\mathbf{0}$ & $\mathbf{+ 1}$ & $\boldsymbol{\alpha}$ \\
Tempo (min) & 70 & 90 & 120 & 150 & 170 \\
Concentração da solução de sacarose ('Brix) & 20 & 30 & 45 & 60 & 70 \\
Proporção fruta:solução & $1: 1,6$ & $1: 3$ & $1: 5$ & $1: 7$ & $1: 8,4$ \\
\hline$\alpha=\left(2^{3}\right)^{1 / 4}=1,68$ & \multicolumn{5}{c}{}
\end{tabular}

Após definida a melhor condição para a desidratação osmótica de laranjas, as amostras foram submetidas à secagem convectiva convencional utilizando-se um secador de bandejas com escoamento paralelo de ar a $60{ }^{\circ} \mathrm{C}$; este processo foi conduzido até que as mesmas atingissem uma faixa de 
umidade intermediária, 20\% (base úmida) caracterizando o produto como "fruta seca", segundo a legislação brasileira (Brasil, 2005). Este teor de umidade foi obtido através do levantamento de uma curva de secagem e acompanhamento da redução de massa durante aproximadamente $18 \mathrm{~h}$ de secagem, sendo avaliados, posteriormente, seus parâmetros físico-químicos.

O teor de umidade das laranjas in natura secas convencionalmente e daquela que receberam o pré-tratamento osmótico antes da secagem, foi determinado em estufa com circulação de ar a $65^{\circ} \mathrm{C}$ até obtenção de massa constante, conforme Eq. 4.

$$
\mathrm{U}=\frac{\left(1-\mathrm{m}_{\mathrm{ss}}\right)}{\mathrm{m}_{\mathrm{u}}} \times 100
$$

em que:

$$
\begin{array}{ll}
\mathrm{U} & \text { - umidade, } \% \\
\mathrm{~m}_{\mathrm{ss}} & \text { - massa de sólido seco, } \mathrm{g} \\
\mathrm{m}_{\mathrm{u}} & \text { - massa de amostra úmida, } \mathrm{g}
\end{array}
$$

Os parâmetros de cor nas amostras foram determinados utilizando-se um colorímetro Minolta. Os valores das coordenadas $\mathrm{a}^{*} \mathrm{e} \mathrm{b}^{*}$ foram utilizados para calcular o ângulo Hue $\left(\alpha^{*}\right)$ e o índice de saturação $\left(\mathrm{C}^{*}\right)$ conforme as Eqs. 5 e 6, respectivamente.

$$
\begin{gathered}
\alpha^{*}=\tan ^{-1} \frac{b}{a} \\
C^{*}=\left(a^{2}+b^{2}\right)^{\frac{1}{2}}
\end{gathered}
$$

Nas laranjas in natura, in natura secas e osmoticamente desidratadas secas, foram realizadas determinações de $\mathrm{pH}$, acidez e vitamina C, conforme AOAC (1995). A acidez titulável foi expressa em porcentagem de ácido cítrico enquanto o teor de vitamina $\mathrm{C}$ foi expresso em $\mathrm{mg}$ ácido ascórbico.

Os carotenoides foram extraídos com acetona e éter de petróleo e determinados espectrofotometricamente a um comprimento de onda de $449 \mathrm{~nm}$, sendo o teor de carotenoides totais expresso em $\mu \mathrm{g}_{\text {criptoxantina }} \mathrm{g}^{-1}$ amostra , conforme Eq. 7 (SouzaSoares et al., 2010).

$$
[\text { criptoxantina }]=\frac{\left(\mathrm{A} \times \mathrm{V} \times 10^{3}\right)}{\mathrm{M} \times \mathrm{A}_{1 \mathrm{~cm}}^{1 \%}}
$$

em que:

[Criptoxantina] - teor de criptoxantina, em $\mu \mathrm{g} \mathrm{g}^{-1}$ amostra

A - absorvância da solução

$\mathrm{V}$ - volume final da solução, $\mathrm{cm}^{3}$

M - massa inicial da amostra, g

$\mathrm{A}^{1 \%}{ }_{1 \mathrm{~cm}}$ - absortividade molar do pigmento (2386)

Os compostos fenólicos foram extraídos com metanol e determinados em espectrofotômetro a $750 \mathrm{~nm}$, através do reagente de Folin Ciocalteau. Os resultados foram expressos em $\mathrm{mg}_{\text {ácido gálico }} \mathrm{g}^{-1}$ amostra , utilizando-se uma curva padrão de ácido gálico (Souza et al., 2010). A atividade antioxidante das amostras foi avaliada pela inativação do radical livre DPPH (1,1 difenil-2-picrihidrazil) a $515 \mathrm{~nm}$ (Rufino et al., 2007). A capacidade de sequestrar o radical livre foi expressa em porcentagem de inibição, conforme a Eq. 8.

$$
\text { \%Inibição }=\frac{\left(\mathrm{A}_{\mathrm{DPPH}}-\mathrm{A}_{\text {amostra }}\right)}{\mathrm{A}_{\mathrm{DPPH}}} \times 100
$$

em que:

$\mathrm{A}_{\mathrm{DPPH}}$ - absorvância da solução de DPPH

$\mathrm{A}_{\text {amostra }}$ - absorvância da solução de DPPH contendo extrato fenólico das amostras

Todos os experimentos foram realizados em triplicata e os resultados foram tratados através da análise de variância (Anova) seguida do teste de Tukey com grau de confiança de 95\%.

\section{Resultados E Discussão}

A Tabela 2 apresenta os valores obtidos para GS, PA e RM, nas diferentes condições estudadas segundo o planejamento experimental. Obtiveram-se valores de GS de 1,2 a 2,4 $\mathrm{g}_{\text {sólidos }} 100 \mathrm{~g}^{-1}$, PA de 4,7 a 7,9 gágua $100 \mathrm{~g}^{-1}$ e RM de 3,6 a 5,5 g $100 \mathrm{~g}^{-1}$. Em todos os experimentos a PA foi maior que o GS o que é importante para o processo tendo em vista que um dos objetivos da desidratação osmótica é a pequena incorporação de sólidos e a grande perda de água.

A Tabela 3 apresenta os efeitos que foram significativos ( $\mathrm{p}<0,05)$ no GS, PA e RM das laranjas e os valores de coeficientes de regressão utilizados para as construções das superfícies de respostas. A concentração de sacarose na solução osmótica foi o fator que mais afetou os GS, PA e RM; para o GS a concentração de sacarose apresentou maior efeito positivo, sinal de que um aumento da concentração da solução de 30 para $60{ }^{\circ}$ Brix aumentou a média da incorporação de sólidos em 1,6 $\mathrm{g}_{\text {sólidos }} 100 \mathrm{~g}^{-1}{ }_{\text {amostra }}$. Um aumento da concentração da solução promoveu aumento na taxa de PA devido ao aumento na pressão osmótica no exterior da fruta. Este mesmo comportamento também foi evidenciado por Lima et al. (2004) que constataram que a cinética de desidratação osmótica de melão foi influenciada pelas condições de processo, notoriamente pela concentração da solução osmótica. Um incremento na interação tempo e na concentração de sacarose, conduziu a um aumento da PA; para a RM a concentração de sacarose (linear) também apresentou efeito positivo enquanto os demais fatores apresentaram efeitos negativos indicando que um incremento nessas variáveis causa uma redução no valor de RM.

Visando à verificação dos modelos matemáticos, realizouse uma análise de variância com as respostas para as variáveis estudadas (Tabela 4). A fim de considerar a possibilidade de que uma variável é preditiva, o valor de $\mathrm{F}$ calculado deve ser de 3 a 5 vezes maior que o $\mathrm{F}_{\text {Fisher }}$ (Schmidt \& Salas-Mellado, 2009); no caso deste trabalho para os GS, PA e RM o valor de F calculado foi 2,6; 6,9 e 4,3 vezes maior que o $F_{\text {Fisher }}$, respectivamente. Considerando que o estudo trata de um processo que envolve 
Tabela 2. Valores de ganho de sólidos (GS), perda de água (PA) e redução de massa (RM) das laranjas submetidas a desidratação osmótica

\begin{tabular}{|c|c|c|c|c|c|c|}
\hline Ensaio & $\begin{array}{l}\text { Tempo } \\
\text { (min) }\end{array}$ & $\begin{array}{c}\text { Concentração } \\
\text { ('Brix) }\end{array}$ & $\begin{array}{c}\text { Proporção } \\
\text { fruta:solução }\end{array}$ & $\begin{array}{c}\text { GS } \\
\left(g_{\text {solidos }} 100 g^{-1}\right)\end{array}$ & $\begin{array}{c}P A \\
\left(g_{\text {agua }} 100 g^{-1}\right)\end{array}$ & $\begin{array}{c}\text { RM } \\
\left(\mathrm{g} 100 \mathrm{~g}^{-1}\right)\end{array}$ \\
\hline 2 & $+1(150)$ & $-1(30)$ & $-1(1: 3)$ & $1,5 \pm 0,2$ & $5,0 \pm 0,70$ & $3,5 \pm 0,6$ \\
\hline 4 & $+1(150)$ & $+1(60)$ & $-1(1: 3)$ & $4,2 \pm 1,1$ & $11,5 \pm 1,10$ & $7,3 \pm 1,0$ \\
\hline 5 & $-1(90)$ & $-1(30)$ & $+1(1: 7)$ & $2,3 \pm 0,4$ & $5,5 \pm 0,40$ & $3,3 \pm 0,6$ \\
\hline 6 & $+1(150)$ & $-1(30)$ & $+1(1: 7)$ & $3,1 \pm 1,1$ & $5,4 \pm 1,10$ & $2,3 \pm 0,2$ \\
\hline 9 & $-\alpha(70)$ & $0(45)^{\prime}$ & $0(1: 5)^{\prime}$ & $1,9 \pm 0,8$ & $8,6 \pm 1,30$ & $6,7 \pm 1,8$ \\
\hline 10 & $+\alpha(170)$ & $0(45)$ & $0(1: 5)$ & $2,7 \pm 0,7$ & $7,8 \pm 0,56$ & $5,1 \pm 1,6$ \\
\hline 11 & $0(120)$ & $-\alpha(20)$ & $0(1: 5)$ & $3,5 \pm 0,1$ & $5,9 \pm 1,00$ & $2,4 \pm 0,9$ \\
\hline 12 & $0(120)$ & $+\alpha(70)$ & $0(1: 5)$ & $4,6 \pm 0,6$ & $10,2 \pm 1,10$ & $5,6 \pm 2,1$ \\
\hline 13 & 0 (120) & $0(45)$ & $-\alpha(1: 1,6)$ & $2,6 \pm 0,3$ & $6,8 \pm 0,60$ & $4,2 \pm 0,4$ \\
\hline 14 & 0 (120) & $0(45)$ & $+\alpha(1: 8,4)$ & $3,5 \pm 0,6$ & $7,5 \pm 0,30$ & $4,1 \pm 0,0$ \\
\hline
\end{tabular}

GS - Ganho de sólidos; PA - Perda de água; RM - Redução de massa. Resultados expressos como média \pm desvio padrão $(\mathrm{n}=3)$

Tabela 3. Efeitos estimados para o GS, PA e RM durante a desidratação osmótica e coeficientes de regressão

\begin{tabular}{|c|c|c|c|c|}
\hline Resposta & Fatores & Efeito & valor $p$ & $\begin{array}{l}\text { Coeficiente } \\
\text { de regressão }\end{array}$ \\
\hline \multirow{8}{*}{$\begin{array}{l}\text { Ganho de sólidos } \\
\left(\mathrm{g}_{\text {solidos }} 100 \mathrm{~g}^{-1}\right)\end{array}$} & Média & 2,34 & 0,0011 & 2,34 \\
\hline & Tempo (L) & 0,77 & 0,0087 & 0,39 \\
\hline & Sacarose (L) & 1,58 & 0,0021 & 0,79 \\
\hline & Sacarose (Q) & 1,20 & 0,0044 & 0,60 \\
\hline & Proporção (L) & 0,86 & 0,0070 & 0,43 \\
\hline & Proporção (Q) & 0,46 & 0,0284 & 0,23 \\
\hline & Tempo x sacarose & 0,38 & 0,0565 & 0,19 \\
\hline & Tempo x proporção & 0,43 & 0,0447 & 0,22 \\
\hline \multirow{5}{*}{$\begin{array}{l}\text { Perda de água } \\
\left(g_{\text {água }} 100 g^{-1}\right)\end{array}$} & Média & 7,75 & 0,0001 & 7,75 \\
\hline & Sacarose (L) & 3,99 & 0,0005 & 2,00 \\
\hline & Proporção $(Q)$ & $-0,46$ & 0,0408 & $-0,23$ \\
\hline & Tempo x sacarose & 0,65 & 0,0301 & 0,33 \\
\hline & Sacarose x proporção & $-1,12$ & 0,0102 & $-0,56$ \\
\hline \multirow{7}{*}{$\begin{array}{l}\text { Redução de } \\
\text { massa } \\
\left(g_{\left.100 g^{-1}\right)}\right.\end{array}$} & Média & 5,40 & 0,0004 & 5,40 \\
\hline & Tempo (L) & $-0,55$ & 0,0293 & $-0,28$ \\
\hline & Sacarose (L) & 2,41 & 0,0016 & 1,21 \\
\hline & Sacarose $(Q)$ & $-1,05$ & 0,0101 & $-0,53$ \\
\hline & Proporção (L) & $-1,01$ & 0,0088 & $-0,51$ \\
\hline & Proporção (Q) & $-0,93$ & 0,0128 & $-0,47$ \\
\hline & Sacarose x proporção & $-0,88$ & 0,0197 & $-0,44$ \\
\hline
\end{tabular}

diversas etapas, os três modelos foram considerados preditivos; portanto, o modelo foi suficientemente confiável para predizer as respostas de qualquer combinação entre as variáveis; além disto, os coeficientes de determinação dos modelos indicaram que, em média, $88 \%$ do comportamento da resposta podem ser explicados pela função estimada (Figura 1); assim os modelos foram adequados para descrever os resultados através das superfícies de resposta, fato importante quando se pretende adotar o processo em maior escala especialmente para uma fruta cujas características indicativas de maturidade são bastante afetadas por variáveis abióticas.

Através da Figura 1A, pode-se constatar que uma incorporação menor de sólidos pela fruta foi obtida em concentrações de solução osmótica entre 30 a $45{ }^{\circ}$ Brix e
Tabela 4. Análise de variância para o ganho de sólidos (GS), perda de água (PA) e redução de massa (RM) da laranja no processo de desidratação osmótica

\begin{tabular}{|c|c|c|c|c|c|c|}
\hline Resposta & $\begin{array}{l}\text { Fonte de } \\
\text { variação }\end{array}$ & SQ & GL & MQ & $\mathrm{F}_{\text {calculado. }}$ & $\mathrm{F}_{\text {Fischer }}$ \\
\hline \multirow{5}{*}{$\begin{array}{c}\mathrm{GS} \\
\left(\mathrm{g}_{\text {solidos }} 100 \mathrm{~g}^{-1}\right)\end{array}$} & Regressẩo & 18,45 & 7 & 2,64 & \multirow[t]{5}{*}{8,58} & \multirow[t]{5}{*}{3,29} \\
\hline & Resíduos & 2,76 & 9 & 0,31 & & \\
\hline & Falta de ajuste & 2,73 & 7 & & & \\
\hline & Erro puro & 0,04 & 2 & & & \\
\hline & Total & 21,21 & 16 & & & \\
\hline \multirow{5}{*}{$\begin{array}{c}\text { PA } \\
\left(g_{\text {água }} 100 g^{-1}\right)\end{array}$} & Regressão & 58,72 & 4 & 14,68 & \multirow[t]{5}{*}{22,36} & \multirow[t]{5}{*}{3,26} \\
\hline & Resíduos & 7,88 & 12 & 0,66 & & \\
\hline & Falta de ajuste & 7,83 & 10 & & & \\
\hline & Erro puro & 0,05 & 2 & & & \\
\hline & Total & 66,60 & 16 & & & \\
\hline \multirow{5}{*}{$\begin{array}{c}\mathrm{RM} \\
\left(\mathrm{g} 100 \mathrm{~g}^{-1}\right)\end{array}$} & Regressão & 31,70 & 6 & 5,28 & \multirow[t]{5}{*}{13,99} & \multirow[t]{5}{*}{3,22} \\
\hline & Resíduos & 3,77 & 10 & 0,37 & & \\
\hline & Falta de ajuste & 3,71 & 8 & & & \\
\hline & Erro puro & 0,06 & 2 & & & \\
\hline & Total & 35,47 & 16 & & & \\
\hline
\end{tabular}

SQ - Soma quadrática; GL - graus de liberdade; $M Q$ - média quadrática; $F$ alluado - valor $F$ calculado para 0 modelo matemático; $F_{\text {Fisher }}$ - valor $F$ de Fischer a um nível de $0,05 \%$ de significância

proporção fruta:solução osmótica de até 1:5. Condições semelhantes foram encontradas por Gonçalves \& Blume (2008) para a desidratação osmótica do abacaxi, e por Shigematsu et al. (2005) quando desidrataram osmoticamente carambolas, frutas com atividade de água semelhante à da laranja.

Com relação à PA, maiores valores foram obtidos quando se utilizaram maiores concentrações da solução de sacarose (Figura 1B). O aumento da concentração da solução de sacarose gerou fluxos de massas que ocasionaram uma variação da concentração da solução e diminuição dos gradientes de concentração entre a fruta e a solução osmótica, ao longo do processo, promovendo um aumento na taxa de perda de água (Dalla \& Giroux, 2001).

A utilização de maiores proporções de solução osmótica minimiza o efeito da diluição da solução aumentando a eficiência da desidratação. Os melhores valores para RM foram 
A.

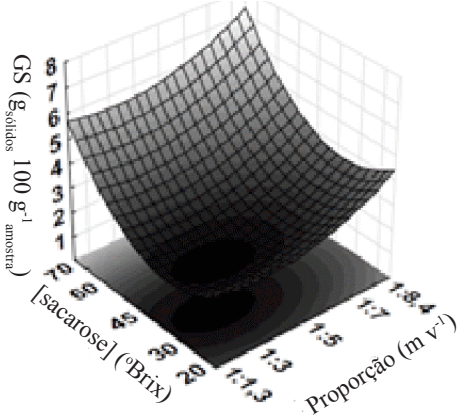

$\mathrm{GS}=2,34+0,39 \mathrm{t}+0,79[\mathrm{sac}]+0,60[\mathrm{sac}]^{2}+$

$+0,43$ prop $+0,23$ prop $^{2}+0,19 t *[\mathrm{sac}]+0,22 \mathrm{t} *$ prop $\mathrm{R}^{2}=87 \%$
B.

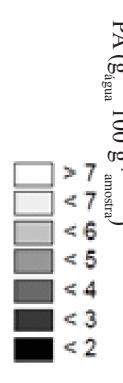
$\mathrm{R}^{2}=88 \%$
C.

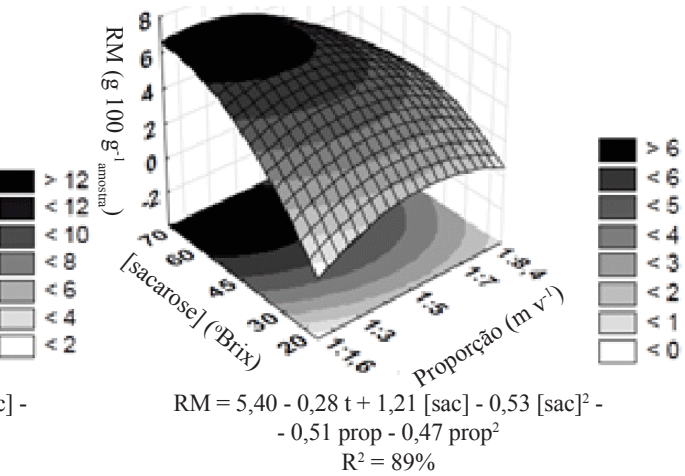

Figura 1. Superfícies de resposta que correlacionam o ganho de sólidos (A), a perda de água (B) e a redução de massa (C) com a concentração de sacarose da solução osmótica e proporção fruta:solução osmótica. Sendo: GS - ganho de sólidos; PA - perda de água; RM - redução de massa; [sac] - concentração de sacarose; prop - proporção fruta solução osmótica; $\mathrm{t}$ - tempo de desidratação osmótica e $\mathrm{R}^{2}$ - coeficiente de determinação

alcançados com concentrações de sacarose de 45 e $60^{\circ}$ Brix e nas proporções de 1:3 e 1:5 fruta:solução osmótica.

O tempo foi a variável que menos afetou o processo e mostrou efeito negativo para a RM; em razão disto, escolheu-se o menor tempo minimizando esses efeitos e contribuindo para a execução do processo. O efeito do tempo na desidratação osmótica pode ser explicado pela modificação do tecido vegetal, principalmente das membranas celulares, que vão enfraquecendo com longos períodos de exposição à solução osmótica (Córdova, 2006). A partir daí, os ensaios 3, 4 e 9 apresentam as melhores condições para o processo de desidratação osmótica; no entanto, por apresentar maior tempo de execução (150 min) o ensaio 4 não foi escolhido e entre os ensaios 3 e 9 aquele que possibilitou melhores resultados para a RM e PA foi o 3 (90 min, $60^{\circ}$ Brix, 1:3); portanto, este foi o escolhido como sendo o mais adequado na desidratação osmótica das laranjas.

Foram avaliadas as perdas de umidade da laranja em cada etapa do processo a fim de verificar e garantir sua eficiência. Inicialmente, a umidade da fruta in natura era de $88,9 \%$; analisando a umidade após a desidratação osmótica, verificouse uma redução de umidade igual a $5,1 \%$, ou seja, a umidade da laranja passou a ser de $83,3 \%$; no processo de secagem convectiva, a redução da umidade foi de $66,4 \%$, atingindo, assim, a umidade final desejada para caracterizar uma fruta seca, em torno de $20 \%$. Depois de submetidas à melhor condição de desidratação osmótica, as laranjas foram secas convencionalmente em secador de bandejas.

A Tabela 5 apresenta os resultados dos parâmetros colorimétricos avaliados nas laranjas in natura, secas e desidratadas osmoticamente utilizando-se solução de sacarose.

A luminosidade se relaciona ao brilho da superfície, sendo representada em uma escala de 0 a 100 , em que os maiores valores indicam maior brilho. Os valores do parâmetro $L^{*}$ indicam que a secagem não reduziu o brilho das amostras significativamente $(\mathrm{p}<0,05)$ após secagem com e sem pré-tratamento osmótico. Analisando o parâmetro $\mathrm{a}^{*}$, todas as amostras apresentaram maior tendência à cor vermelha enquanto para $b^{*}$ as amostras tenderam para a cor amarela, possivelmente pela presença de carotenoides. Os valores de $b^{*}$ das amostras secas diferiram $(\mathrm{p}<0,05)$ das amostras in natura, embora tenham ocorrido perdas de carotenoides; durante o processamento (Tabela 6) as amostras secas apresentaram maior tendência à cor amarela do que a fruta in natura, fato que pode estar relacionado ao início da caramelização das amostras durante o processo de secagem.

Tabela 6. Características físico-químicas das laranjas in natura, in natura seca e desidratada osmoticamente e seca

\begin{tabular}{|c|c|c|c|}
\hline \multirow{2}{*}{$\begin{array}{l}\text { Propriedades } \\
\text { físico-químicas }\end{array}$} & \multicolumn{3}{|c|}{ Laranjas } \\
\hline & IN & $\mathbf{I N}_{\text {seca }}$ & $\mathrm{DO}_{\text {seca }}$ \\
\hline $\mathrm{pH}$ & $3,9 \pm 0,1 \mathrm{a}$ & $3,9 \pm 0,3 a$ & $3,7 \pm 0,6 \mathrm{a}$ \\
\hline Acidez Titulável (\% ác. cítrico)* & $6,8 \pm 0,3 a$ & $4,9 \pm 0,1 b$ & $2,7 \pm 0,0 \mathrm{c}$ \\
\hline Vitamina C (mgác. ascórbico $\left.100 \mathrm{~g}^{-1}\right)^{*}$ & $415,0 \pm 0,3 a$ & $31,5 \pm 0,3 c$ & $37,3 \pm 0,1 b$ \\
\hline Carotenóides $\left(\mu \mathrm{g}_{\text {criptoxantina }} \mathrm{g}^{-1}\right)^{*}$ & $35,0 \pm 0,9 a$ & $2,7 \pm 0,5 b$ & $3,9 \pm 0,0 b$ \\
\hline Fenóis Totais $\left(\mathrm{mg}_{\text {AC. gálico }} \mathrm{g}^{-1}\right)^{\star}$ & $5,1 \pm 0,0 \mathrm{a}$ & $1,7 \pm 0,3 \mathrm{c}$ & $4,0 \pm 0,2 b$ \\
\hline Atividade antioxidante (\% Inibição) & ) $91,3 \pm 1,0 a$ & $42,3 \pm 2,1 \mathrm{c}$ & $76,3 \pm 4,6 b$ \\
\hline
\end{tabular}

Quanto ao ângulo de Hue, em todas as amostras os valores foram próximos de 90 graus reforçando a tendência para a tonalidade amarela. Os valores de $\mathrm{C}^{*}$ menores correspondem ao padrão de cor mais fraco (aspectos fosco do objeto) e valores mais altos ao padrão de cor mais intensa, aspecto desejado para os alimentos (Cardoso et al., 2007). Constatou-se que amostras

Tabela 5. Parâmetros de cor das laranjas in natura e secas

\begin{tabular}{cccccc}
\hline Amostra de laranja & $\mathbf{L}^{*}$ & $\mathbf{a}^{*}$ & $\mathbf{b}^{\boldsymbol{*}}$ & $\mathbf{a}^{*}$ & $\mathbf{C}^{*}$ \\
in natura & $47,1 \pm 1,0 \mathrm{a}$ & $6,0 \pm 0,8 \mathrm{~b}$ & $28,6 \pm 0,2 \mathrm{~b}$ & $78,1 \pm 0,1 \mathrm{~b}$ & $29,3 \pm 0,3 \mathrm{c}$ \\
in natura seca & $45,4 \pm 1,2 \mathrm{a}$ & $8,5 \pm 1,1 \mathrm{a}$ & $42,2 \pm 1,2 \mathrm{a}$ & $78,6 \pm 0,7 \mathrm{ab}$ & $43,1 \pm 0,8 \mathrm{a}$ \\
D0 seca & $45,9 \pm 1,3 \mathrm{a}$ & $7,3 \pm 0,9 \mathrm{ab}$ & $39,8 \pm 1,3 \mathrm{a}$ & $79,6 \pm 0,6 \mathrm{a}$ & $40,5 \pm 0,5 \mathrm{~b}$ \\
\hline
\end{tabular}

DO - desidratada osmoticamente; $L^{*}$ - luminosidade; $a^{*}$ - croma $a ; b^{*}$ - croma $b ; \alpha^{*}$ - ângulo de Hue; $C^{*}$ - índice de saturação. Letras iguais seguidas das médias não diferem estatisticamente quanto às diferentes amostras (Teste de Tukey, $p<0,05$ ) 
secas apresentaram maiores índices de saturação indicando que elas apresentaram cores mais fortes em virtude do aumento na concentração de sólidos. Segundo Sanjinez-Argandoña et al. (2004) o efeito benéfico e protetor do pré-tratamento osmótico na retenção da cor do produto osmo-desidratado e seco demonstra a preferência dos consumidores; o efeito da desidratação osmótica como pré-tratamento à secagem nas propriedades físico-químicas das amostras, está apresentado na Tabela 6.

$\mathrm{O}$ valor de $\mathrm{pH}$ da laranja in natura está de acordo com o reportado em outros trabalhos (Branco et al., 2007; Zangelmi et al., 1996) não se observando diferença significativa ( $p$ $<0,05)$ de $\mathrm{pH}$ com as amostras que foram secas em razão, possivelmente, do forte poder tamponante do ácido cítrico e da menor atividade de água proporcionada pela secagem. Aspectos foram reforçados pelos teores de acidez, em que todas as amostras diferiram $(\mathrm{p}<0,05)$ entre si. A perda de ácido cítrico nas amostras desidratadas osmoticamente sugere que houve migração do ácido para as soluções osmóticas durante o processo de desidratação.

$\mathrm{O}$ teor de vitamina $\mathrm{C}$ em todas as amostras secas diminuiu quando comparado ao da fruta in natura; contudo, para as amostras tratadas osmoticamente a perda no teor de ácido ascórbico foi menor que naquelas frutas que não passaram por este pré-tratamento. Além do processamento térmico a agitação mecânica da laranja propicia a redução do teor da vitamina $\mathrm{C}$ uma vez que, durante o processo de agitação, há incorporação de oxigênio com consequente oxidação dessa vitamina (Fernandes et al., 2007).

A secagem também promoveu uma redução acentuada nos teores de carotenoides, mas nas laranjas previamente desidratadas as perdas foram menores. Esta perda no teor de carotenoides é atribuída à temperatura de secagem visto que esses também são sensíveis à luz e acidez, bem como reações de oxidação, que podem ser acentuadas pela temperatura de secagem.

O conteúdo de fenois encontrado para a laranja in natura foi semelhante ao reportado na literatura (Oliveira et al., 2007). Todas as amostras diferiram estatisticamente em relação ao conteúdo de compostos fenólicos sendo que as laranjas prédesidratadas osmoticamente apresentaram as menores perdas em relação à fruta in natura seca, cerca de $22 \%$ enquanto as laranjas secas sem pré-tratamento apresentaram perda elevada desses compostos (cerca de 67\%). Portanto o pré-tratamento osmótico foi eficaz na retenção dos compostos fenólicos presentes na laranja.

Os extratos das amostras das laranjas in natura e desidratadas exibiram percentual de sequestro do radical livre DPPH acima de $70 \%$, ou seja, uma forte capacidade antioxidante o que confere, ao produto, uma importante característica pois tais antioxidantes auxiliam na prevenção de doenças degenerativas e cardiovasculares e beneficiam a própria conservação do produto. Variações na capacidade antioxidante de diferentes extratos podem ser atribuídas à diferença na composição química tais como fenólicos, ácido ascórbico e carotenoides, justificando os resultados obtidos para o potencial antioxidante preservado pela desidratação osmótica (Couto \& CanniattiBrazaca, 2010).
Os resultados indicaram que a operação de desidratação osmótica que antecedente a secagem convencional, é uma alternativa promissora pois, além de não acrescentar elevados custos ao processo, permite a elaboração de um produto alimentício com atividade antioxidante preservada e com maior facilidade de consumo.

\section{ConclusõES}

1. Menores valores de ganho de sólidos e maiores de perda de água e redução de massa foram obtidos em laranjas desidratadas osmoticamente em menores níveis de tempo e de proporção fruta:solução e maiores níveis de concentração de solução osmótica.

2. A melhor condição para a desidratação osmótica foi obtida utilizando-se uma solução de sacarose de $60^{\circ}$ Brix, na proporção fruta:solução de 1:3 durante $90 \mathrm{~min}$.

3. O processo de secagem convencional reduziu os teores de carotenoides e de vitamina $\mathrm{C}$ mesmo utilizando um prétratamento osmótico enquanto as perdas no conteúdo fenólico e na atividade antioxidante das laranjas secas foram minimizadas em 45,1 e 37,3\% com a desidratação osmótica.

\section{Literatura Citada}

AOAC - Association of Official Analytical Chemists. Official methods of analysis, Ch.27, p.6; Ch.37, p.10; Ch.45, p.16, Washinton: AOAC, 1995.

Branco, I. G.; Sanjinez-Argandoña, E. J.; Silva, M. M.; Paula, T. M. Avaliação sensorial e estabilidade físico-química de um blend de laranja e cenoura. Ciência e Tecnologia de Alimentos, v.27, p.7-12, 2007.

Brasil. Agência Nacional de Vigilância Sanitária - Regulamento técnico para produtos de vegetais, produtos de frutas e cogumelos comestíveis. Resolução $\mathrm{RDC} \mathrm{n}^{\circ} 272$, de 22 de setembro de 2005.

Cardoso, W. S.; Pinheiro, F. A.; Patelli, T.; Perez, R.; Ramos, A. M. Determinação da concentração de sulfito para a manutenção da qualidade da cor em maçã desidratada. Revista Analytica, v.29, p.66-72, 2007.

Carvalho, P.; Machado, C.; Moretti, C.; Fonseca, E. Hortaliças como alimentos funcionais. Revista Horticultura Brasileira, v.24, p.397-404, 2006.

CONAB - Companhia Nacional de Abastecimento - Acompanhamento da Safra de Laranja2011/12.http://www.conab. gov.br/OlalaCMS/uploads/arquivos/12_05_17_17_48_56_ boletim_laranja_dez_2011.pdf. 17 Set. 2012.

Córdova, K. R. Desidratação osmótica e secagem convectiva de maçã Fuji comercial e industrial. Curitiba: UFPR, 2006. 148p. Dissertação Mestrado

Couto, M. A.; Canniatti-Brazaca, S. G. Quantificação de vitamina $C$ e capacidade antioxidante de variedades cítricas. Ciência e Tecnologia de Alimentos, v.30, p.15-19, 2010.

Dalla, R. M.; Giroux, F. Osmotic treatments and problems related to the solution management. Journal of Food Engineering, v.49, p.223-236, 2001. 
Demczuk Júnior, B.; Fachin, D. T.; Ribani, R. H.; Freitas, R. J. S. Degradação da cor e do ácido ascórbico na desidratação osmótica de kiwi. Boletim do Centro de Pesquisa e Processamento de Alimentos, v.26, p.229238, 2008.

Dionello, R. G.; Berbert, P. A.; Molina, M. A. B.; Pereira, R. C.; Viana, A. P.; Vinicius, O.; Carlesso, V. O. Desidratação osmótica de frutos de duas cultivares de abacaxi em xarope de açúcar invertido. Revista Brasileira de Engenharia Agrícola e Ambiental, v.13, p.596-605, 2009.

Eren, I.; Kaymak-Ertekin, F. Optimization of osmotic dehydration of potato using response surface methodology. Journal of Food Engineering, v.79, p.344-352, 2007.

Fernandes, A. G.; Maia, G. A.; Souza, P. H. M.; Costa, J. M. C.; Figueiredo, R. W.; Prado, G. M. Comparação dos teores em vitamina $\mathrm{C}$, carotenóides totais, antocianinas totais e fenólicos do suco tropical de goiaba nas diferentes etapas de produção e influência da armazenagem. Alimentos e Nutrição, v.18, p.431-438, 2007.

Gonçalves, A. A.; Blume, A. R. Efeito da desidratação osmótica como tratamento preliminar na secagem do abacaxi. Estudos Tecnológicos, v.4, p.124-134, 2008.

Lima, A. S.; Figueiredo, R. W.; Maia, G. A.; Lima, J. R.; Souza Neto, M.; Souza, A. C. R. Estudo das variáveis de processo sobre a cinética de desidratação osmótica de melão. Ciência e Tecnologia de Alimentos, v.24, p.282286, 2004.

Martim, N. S. P. P.; Waszczynskyj, N.; Masson, M. L. Cálculo das variáveis na desidratação osmótica de manga cv. Tommy Atkins. Ciência e Agrotecnologia, v.31, p.1755-1759, 2007.

Medeiros, C. D.; Cavalcante, J. A.; Alsina, O. L. Estudo da desidratação osmótica da fruta de palma (figo da índia). Revista Brasileira de Produtos Agroindustriais, v.8, p.153$162,2006$.

Oliveira, M. S.; Dors, G. C.; Souza-Soares, L. A.; BadialeFurlong, E. Antioxidant activity of phenolic compounds from plant extracts. Alimentos e Nutrição, v.18, p.267-275, 2007.

Rodrigues, L. K.; Pereira, L. M.; Ferrari, C. C.; Sarantópoulos, C. I. L.; Hubinger, M. D. Vida útil de fatias de manga armazenadas em embalagem com atmosfera modificada passiva. Ciência e Tecnologia de Alimentos, v.28, p.271278, 2008.
Rufino, M. S. M.; Alves, R. E.; Brito, E. S.; Morais, S. M.; Sampaio, C. G.; Péres-Jiménez, J.; Saura-Calixto, F. D. Metodologia científica: Determinação da atividade antioxidante total em frutas pela captura do radical livre DPPH. Fortaleza: Ministério da Agricultura, Pecuária e Abastecimento, 2007. 4p. Comunicado Técnico 127.

Ruiz-López, I. I.; Ruiz-Espinosa, H.; Herman-Lara, E.; Zárate-Castillo, G. Modeling of kinetics, equilibrium and distribution data ofosmotically dehydration carambola (Averrhoa carambola L.) in sugar solutions. Journal of Food Engineering, v.104, p.218-226, 2011.

Sanjinez-Argandoña, E. J.; Menegalli, F. C.; Cunha, R. L.; Hubinger, M. D.Comparação da cinética de desidratação osmótica de goiabas em sistema de bancada esemicontínuo. Revista de Ciências Exatas e Naturais, v.6, p.57-66, 2004.

Schmidt, C. G.; Salas-Mellado, M. Influência da ação das enzimas Alcalase e Flavourzyme no grau de hidrólise das proteínas de carne de frango. Química Nova, v.32, p.1144-1150, 2009.

Shigematsu, E.; Eik, N. M.; Kimura, M.; Mauro, M. A. Influência de pré-tratamentos sobre a desidratação osmótica de carambolas. Ciência e Tecnologia, v.25, p.536-545, 2005.

Souza, M. M.; Oliveira, M. S.; Rocha, M.; Badiale-Furlong, E. Avaliação da atividade antifúngica de extratos fenólicos de cebola, farelo de arroz e microalga Chlorella phyrenoidosa. Ciência e Tecnologia de Alimentos, v.30, p.680-685, 2010.

Souza-Soares, L. A.; Badiale-Furlong, E.; Feddern, V.; Cipolatti, E.; Mendes, G. L.; Giacobbo, G.; Kupski, L.; Oliveira, M. S.; Rocha, M.; Souza, M.; Pinto, S. S. Bioquímica experimental - Uma introdução. Pelotas: Universitária, 2010. 269p.

Tazima, Z. H.; Auler, P. A. M.; Neves, C. S. V. J.; Yada, I. F. U.; Leite Júnior, R. P. Comportamento de clones de laranja Valência na região norte do Paraná, Revista Brasileira de Fruticultura, v.30, p.970-974, 2008.

Tsamo, C. V.; Bilame, A.; Ndjouenkeu, R.; Nono, Y. J. Study of material transfer during osmotic dehydration of onion slices (Allium cepa) and tomato fruits (Lycopersicon esculentum). Food Science and Technology, v.38, p.494-500, 2005.

Zangelmi, A. C. B.; Sgarbieri, V. C.; Garrutti, R. S.; Rodriguez, O.; Verlêngia, F.; Menezes, T. J. B.; Jorge, J. P. N.; Figueiredo, I. B.; Marsaioli, A. Estudo comparativo de diversas variedades paulistas de laranja na produção de suco concentrado. Coletânea do Instituto de Tecnologia de Alimentos, v.1, p.323-363, 1996. 